\title{
EFFECTS OF EXTRINSIC ELECTRIC CURRENT ON THE CYPRINID FISH EIRG (S-POTENTIAL)
}

\author{
KOSUKE WATANABE, TSUNEO TOSAKA \\ AND TSUNEO YOKOTA \\ Department of Physiology, Tokyo Women's Medical College
}

There have been many discussions on the origin of a sustained negative action potential evoked by illumination within the fish retina, which is known as Svaetichin's "cone action potential" (9) and also as "fish EIRG" (intraretinal action potential) circumspectively termed by Tomita (10). Recently trials have been made to clarify the accurate allocation of microelectrode tip, making a visible mark inside the retina with various techniques $(5,7,8,12)$. Their results, though being slightly different in detailed points, show that there is no disagreement with the view of Tomita and his colleagues that the characteristic action potential does not originate in the receptor cells but in a proximal layer to the receptors $(10,11,12,13)$. It has, however, to be pointed out that, although the activities of bipolar and/or horizontal cells instead of receptors are simply assigned to generation of this negative action potential ( $S$-potential), it remains still in obscurity whether it is of intra-or extracellular nature. As was discussed in a previous paper, it appears to be evident that, even if it is of extracellular nature, the recording must be made from within some kind of compartments isolated electrically from each other (11).

The present paper will deal with some electric properties of the cell or the very compartment which gives $S$-potential in response to light, especially with effective resistance as revealed by passing extrinsic electric current through the micropipette placed inside them.

\section{MATERIALS AND METHOD}

Procedure to prepare the experimental materials and the optical assembly employed are essentially similar to those described in a preceding paper (13). The only exception was that the inverted retina $(R)$ was mounted on a small transparent polystylene plate which was fixed at the center of a cork block placed inside a Ringer bath $(V)$ as shown in fig. 1 . This is for the purpose of carrying out the application of electric current under conditions close to that of a volume conductor. Since, however, it was difficult to determine whether or not the tip of the microelectrode did reach just the surface of retina dipped completely in a solution, the level of oxygenated Ringer solution was so adjusted that the retina was half immersed. In the figure is also shown the circuit diagram which consists of two head amplifiers, one for recording of potential led from one barrel of a double-barrelled microelectrode $(E)$ and

Received for Publication September 5, 1959.

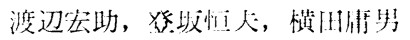




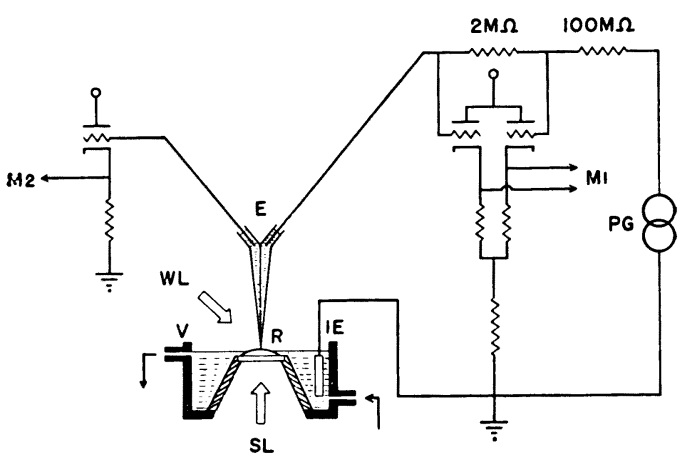

FIG. 1. Schematic illustration of the assembly for recording and application of electric current and the mounting of the retina. See Text.

the other for that of current passed through another barrel from a pulse generator $(P G)$ to the indifferent grounded electrode $(I E)$. As seen from the figure, current is differentially measured as a potential difference across a fixed resistor of $2 \mathrm{M} \Omega$. Being led to the respective main amplifiers $\left(M_{1}, M_{2}\right)$ both current and potential are simultaneously displayed on a cathode-ray oscilloscope. The double-barrelled microelectrodes filled with $3 \mathrm{M} \mathrm{KCl}$ were prepared by Tomita's method, among which those with electric resistance of $10-20 \mathrm{M} \Omega$ and with coupling resistance of $50-200 \mathrm{~K} \Omega$ were selectively used. Values of the latter were measured in Ringer solution before and after each series of experiment, and subtracted from the total resistance to obtain the effective resistance of a certain element.

\section{RESULTS}

The so-called membrane potential and action potential. It is well known that the characteristic $S$-potential is obtained when the microelectrode is advanced and a sudden drop of resting potential appears. Values of the resting potential are variously ranged from below $10 \mathrm{mV}$ to over $30 \mathrm{mV}$. In the present experiments, after adjusting the electrode to be located at a position from where the largest response was recorded, application of current was made. One of such experiments is shown in fig. $2 a$, in which the left hand column indicates the effect of depolarizing current (electrode positive) and the right hand that of hyperpolarization (electrode negative). During the passage of current of about one second duration, the retina was stimulated with white light indicated by small positive (on) and negative (off) pips. In each recording, upper trace represents the current applied and lower the action potential evoked by illumination. The control response is shown in fig. $2 b$. As seen from the figure, it is evident that the response does not show any essential change in its size and configuration even when the so-called membrane potential is shifted about $15 \mathrm{mV}$ towards both sides of the resting level. Changes in membrane potential brought by current applied appears to be represented as a linear function of the latter. The calculated effective resistance was 1.5 megohms in this case. These relations were minutely illustrated in figs. 3 and 4. 


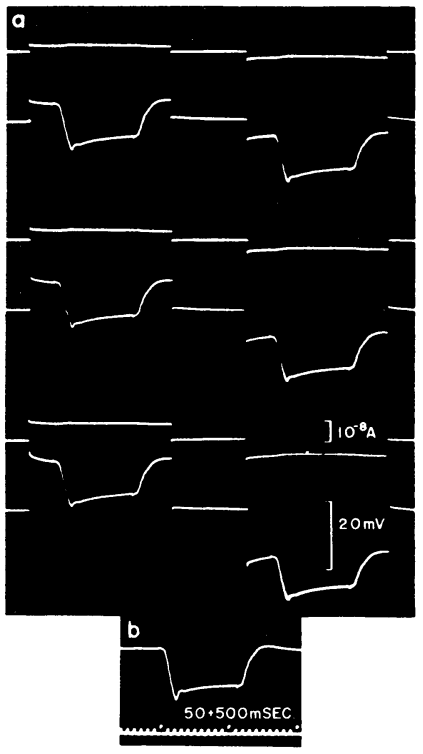

FIG. 2. Effect of current passage on resting and active states. ( $a$ ) Electric current applied (upper trace in each recording) and action potentials evoked by illumination (lower). (b) Control response without application of electric current. See text.

In fig. 3 is shown another example of the similar experiments, in which the sweeping speed was reduced in order to observe the successive responses under a series of current application. Record $A$ indicates the currents and $B$ the responses. The first and the last record. ings in $B$ are the controls obtained without current application. Comparing with the first action potential, the last one shows a slight deformation in its offresponse. However, this is first found in the third response which was evoked during the passage of weak hyperpolarizing current, and appears not to be a significant change produced by the current but to be caused by insertion of the electrode. In this experiment, the current intensity was increased stepwise from $10^{-10}$ to $10^{-8} \mathrm{~A}$ in sequence indicated in the figure, only records taken under the current of less than $10^{-9}$ A were not shown. Again it is clear that neither depolarization nor hyperpolarization gives the slightest influence to the original response to light.

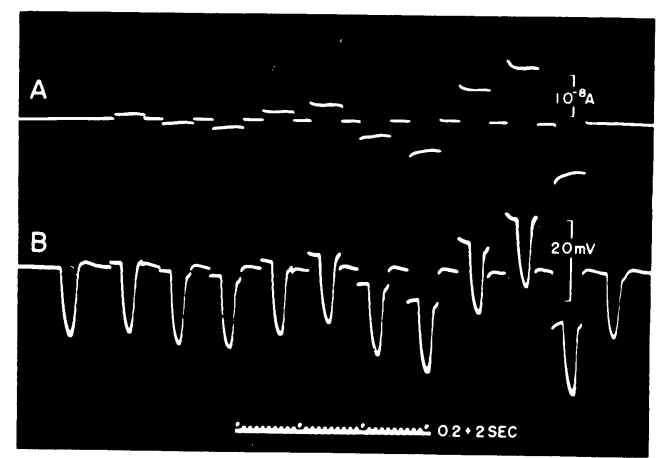

FIG. 3. Effect of current passage on resting and active states. ( $A$ ) A series of current pulses applied. (B) $S$-potentials evoked by illumination during current application. The first and the last responses are con. trols. See text. 
Fig. 4, taken from the experiment shown in fig. 3, illustrates the relationship between current intensity and membrane potential shifted from the resting level of about $-20 \mathrm{mV}$. Both current and potential are represented in logarithmic scale. It must be pointed that there exists direct proportionality between current and membrane potential manifested by a straight line extending over a wide value of membrane potential, and, especially, that there can be observed neither rectification property nor specific level in any sense as usually seen in an excitatory cell membrane. This means that the very membrane, from inside which the sustained negative action potential can be recorded, is of completely passive nature against electric current. The effective resistance calculated is about $1 \mathrm{M} \Omega$.

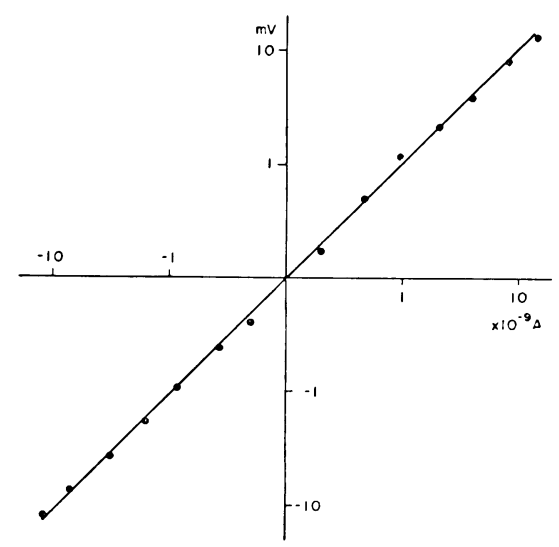

FIG. 4. Relation between electric current applied and membrane potential shifted from the resting level. Taken from fig. 3. See text.

Application of the repetitive electric current pulses during resting and active states. From the above results, it is expected that the so-called membrane resistance will be unchanged not only in resting state but also during eliciting action potential. To examine this presumption, repetitive short rectangular pulses were employed instead of single D. C. pulses in the following experiments. In fig. 5 is shown one of the series of such experiments. Frequency of pulses applied was about twenty per second, and the duration of a single pulse, taken to be equal to that of the following pause, was about $25 \mathrm{msec}$. Column $A$ indicates the effect of positive pulses and $B$ that of negative ones. The top recordings in $a$ are currents applied. As seen from record $a$, in which each response is divided into two similar figures by rectangular pulses, it is clear that the effective resistance is not affected by illumination. This is more evidently shown in records $c$ and $d$ indicating the falling and rising phases respectively under faster display. In record $b$, the intensity of stimulating light was decreased to one-tenth that introduced in $a$ under the same condition as to the application of current. Again in this experiment, it is evident that the shift of the membrane potential produced by current passage is unchanged during illumination. In other words, the electric property of the 


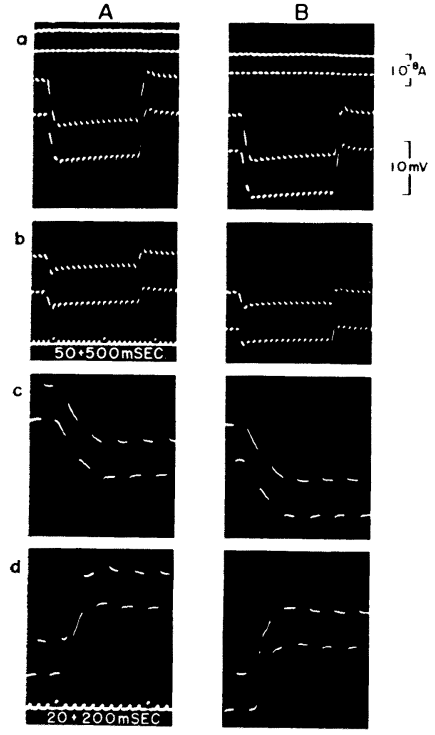

membrane is quite independent of light stimulus which naturally affects the amplitude of response. The effective resistance of $1.6 \mathrm{M} \Omega$ was obtained in this case.

FIG. 5. Effect of positive $(A)$ and negative $(B)$ repetitive rectangular pulses on resting and active states. The top traces in $(a)$, currents. In $(b)$, intensity of stimulating light was reduced to one-tenth that in $(a) .(c),(d)$ Falling $(c)$ and rising $(d)$ phases of $S$-potential under faster display. See text.

Spectral response pattern and effective resistance. Although all the recordings presented above are of L-elements in response to spectral illumination, it has been found that there is no difference between L-and RG-elements in their manners against the current passage. An example of RG-elements is shown in fig. $6 A$ as compared with L-element presented in $B$. In the figure, original responses to white light are shown in record $a$ and their spectral response patterns in $b$. Records $c$ and $d$ illustrate the responses during the application of positive and negative pulses respectively. Magnitude of currents are indicated by short bars at the right edge in $A$. Pulse frequency is about twice that employed in fig. 5. It should be noticed that record $A$, except its spectral response pattern, has strong resemblance to record $B$, only the shift in membrane potential brought by the current of equal intensity is considerably larger in RG-element than in L-element. Especially, it must be pointed out that their original responses appear to be qualitatively similar to each other in configuration. In other words, it appears that there is not a marked interrelation between the spectral response and the response to white light concerning the configuration of the latter.

In the course of the present study, about two hundreds elements were examined with respect to the electric properties of the available membrane. Although three kinds of spectral response pattern, L-, RG-and RYB-types are known in Cyprinus auratus (13), unfortunately RYB-element (triphasic colour response) was not encountered during the present experiment. The distribution of the values of the effective resistance obtained is shown in fig. $\tau$, in which abscissa indicates the values of the effective resistance in megohms and ordinate number of the elements in percentage, taking the maximal number as $100 \%$. Open blocks represent L-elements and hatched blocks RG-elements. 

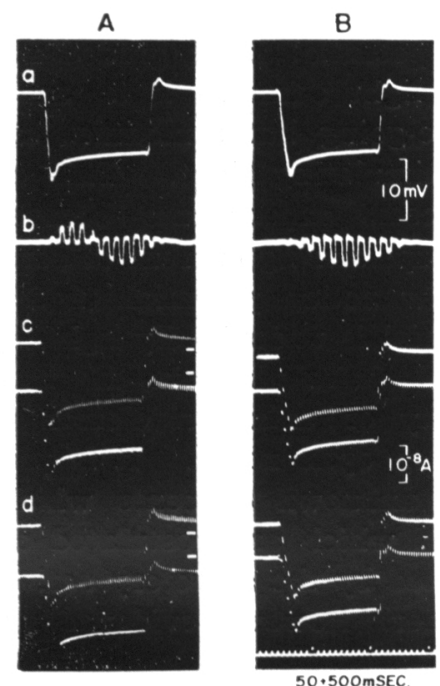

FIG. 6

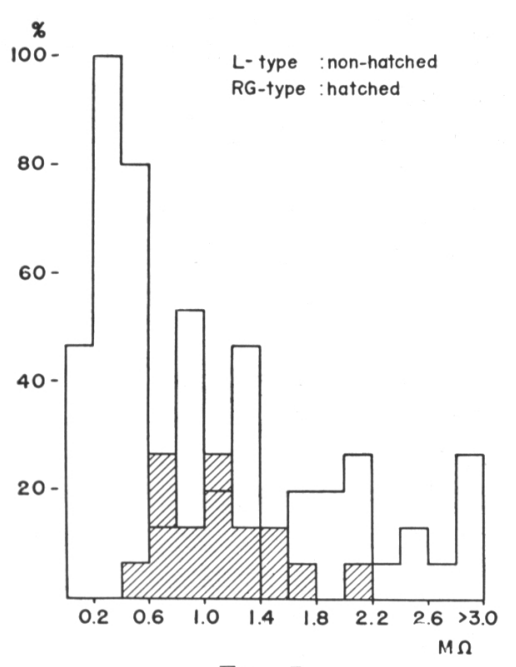

FIG. 7

FIG. 6. Comparison between RG-(A) and L-(B) elements. (a) Original responses to white light. (b) Spectral response patterns. (c), (d) Application of positive $(c)$ and negative $(d)$ repetitive current pulses. See text.

FIG. 7. Distribution of the values of effective resistance. Hatched blocks, RGelements, and open blocks, L-elements. See text.

As seen from the figure, the values of the effective resistance are distributed in a wide range from below $100 \mathrm{~K} \Omega$ to over $3 \mathrm{M} \Omega$. However, it is noticed that the effective resistance of L-elements is most densely populated in number between the values of $200 \mathrm{~K} \Omega$ and $600 \mathrm{~K} \Omega$, while that of RG-elements is concentrated on the values from $600 \mathrm{~K} \Omega$ to $1.6 \mathrm{M} \Omega$. Considering evidence that RG-responses were almost always recorded from locations about $35 \mu$ proximal (one rough step of the micromanipulator) to the L-elements, difference of the above values of the effective resistance between L- and RG-elements appears to be of interest. MacNichol and Svaetichin has postulated the giant horizontal cell and the bipolar cell to produce the monophasic L-response and the diphasic RG- or YB-response respectively (6). If these action potentials are truely of intracellular nature, and if these cells are assumed to have similar specific membrane resistance, the effective resistance of $600 \mathrm{~K} \Omega-1.6 \mathrm{M} \Omega$, that is apparently comparable to that of motoneurones (1), might be attributed to bipolar cells and that of smaller values to larger horizontal cells. However, these points will be further discussed in relation to the electrical nature of the very responses.

\section{DISCUSSIONS}

Electrical passivity of the membrane and the nature of S-potential. According to Grundfest's classification of electrogenesis (2), it is easily expected that 
the membrane generating the characteristic $S$-potential is electrically inexcitable. This follows from evidence that the potential is of graded nature, being prolonged and.sustained as long as the light stimulus lasts, and that the polarity of the response is usually negative-going but sometimes can be reversed as the change in wavelength of the stimulating light. This expectation appears to be directly proved in the present experiments, because, as mentioned above, neither depolarizing nor hyperpolarizing current of considerable intensity and duration can elicit the slow action potential (figs. 2, 3). The only adequate stimulus to produce $S$-potential is undoubtedly light, by the arrival of it some photochemical reaction should take place in the receptor cells and, after through transmissional activity of unknown elements, the response is finally represented as spike volleys in optic nerve. It is presumable, as suggested by Grundfest (3), that these unknown elements which are interposed between cones and ganglion cells and which are postulated as the second neurones producing $S$-potential are excited by the secretory activity of cones and respond by their own secretory manner. However, evidence is given in the present experiment that the amplitude and configuration of S-potential are quite independent of the change in membrane potential caused by current passage (figs. 2, 3). From this, it must be pointed out that, because of having no equilibrium potential, S-potential is quite different from other electrically inexcitable p.s.p.'s. Furthermore, the very membrane from inside which the recording was made appears to be completely passive rather than inexcitable against electric current, and the membrane does not change its effective resistance during action potential is recorded (figs. 5, 6). In other words, even a part of the membrane itself does not possess e.m.f. of $S$-potential unless the e.m.f. is of special nature never affected by ionic current. Lundberg (4) has reported a negative-going secretory potential of sublingual gland cells in cat which appeared almost unchanged within a large range of membrane potential, and he postulated active transport of $\mathrm{Cl}^{-}$as to its generation. Even, however, in such a type of transport of a certain species of ion, it appears hard to consider that the ion transport is completely unaffected by a certain direction of extrinsic electric current. Furthermore, comparing with the secretory potential of gland cells, there are some difficulties to explain the short latency and the fast time course of falling and rising phases of $S$-potential.

On the other hand, evidences suggesting that $S$-potential is recorded not from inside a cell but from some compartment surrounded with resistive membrane have been discussed previously (11). According to Tomita's observation (10), using a coaxial pencil-type microelectrode with internal electrode $50 \mu$ protruded from the tip of external pipette, two action potentials of almost similar size were simultaneously obtained from a single element. After $35 \mu$ withdrawal of the pencil-electrode, the potential from the external pipette was weakened but still obtainable. This implies that, if the recording is made intracellularly, diameter of the cell must be at least over than $80 \mu$. However, there has been found no such a large cell in the cyprinid fish retina.

In the present study membrane time constant was not investigated on account of technical difficulty. Nevertheless, it appears that the membrane, 
unlike that usually producing p.s.p.'s, is completely inactive as if it were a simple resistive membrane. One of the additional experiments supporting this view is shown in fig. 8. A superfine microelectrode was carefully advanced from receptor side of the retina with each step of less than $20 \mu$. Column $A$ indicates a series of recordings obtained when the microelectrode was advanced and column $B$ those when withdrawing the electrode. Recordings in both columns are corresponding to each other as to the depth of recording electrode. As seen from the figure, the ERG at the surface of the retina, $a$, was gradually changed as the electrode advanced. The positive on-response and following sustained potential decreased gradually in amplitude, and then reversed in polarity at a certain depth, and the negativity grew until to give rise to a large $S$-potential. The bottom record $i$ in column $A$ was obtained at a depth of $140 \mu$. Immediately after it was recorded, the small negative response grew up spontaneously to a large typical $S$-potential shown at the bottom in column $B$. This phenomenon appears to be caused by a slight displacement of the microelectrode tip across some resistive layer, from inside and outside which a large $S$-potential and a smaller minature image of the former would be recorded respectively. Thus, again in this experiment, it is clear that the membrane which gives $S$-potential is only a passive and resistive one and is quite different from usual cell membrane. In addition, it is noticed that a

$A$

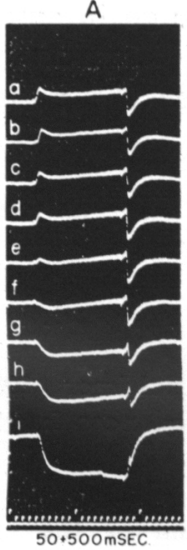

$B$

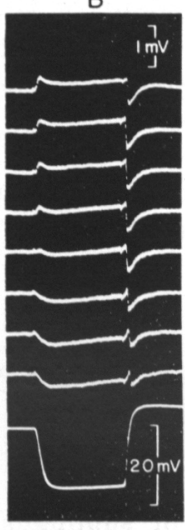
small spike-like off-response remains still in record $i$. This means that the spike-like potential would originate at a depth further proximal to the location from where $S$ potential was recorded.

FIG. 8. Gradual changes in response to white light produced by advancing $(A)$ and withdrawing $(B)$ a superfine microelectrode. See text.

From these observations described above, the very membrane appears to be hardly considered as a cell membrane in its nature. If, however, there is some special mechanism to produce action potential of which the electromotive force is quite independent of extrinsic current passage, and furthermore, if such a mechanism can exist in an electrically passive membrane, it can be said that $S$-potential is being recorded intracellularly. At the present stage, however, it is hard to conclude that this action potential is of intracellular nature.

From the standpoint that it is of extracellular nature, a hypothetical ex- 
planation assuming the existence of some special structure in the cyprinid fish retina will be presented below. If a small active part of a certain cell is inserted into a compartment surrounded with a simple resistive membrane, electric current will flow a wide inactive cell surface to the small sink across the membrane of the compartment. In such a case, even if direct intracellular recording is unable, an electrode located inside the compartment will be able to record a strong negativity compared with the outside of it. Polarity of recorded potential will be reversed if the small sink will be changed to a source.

\section{SUMMARY}

1) For the purpose of clarifying the nature of the so-called cone action potential ( $S$-potential), some electric properties of the membrane, from inside which this action potential was recorded, was investigated by means of doublebarrelled microelectrode technique.

2) Neither depolarizing nor hyperpolarizing current passage gave influence to the original response in its amplitude and configuration. In other words, $S$-potential did not have an equilibrium potential.

3 ) While the values of effective resistance were widely distributed in a range from below $200 \mathrm{~K} \Omega$ to over $3 \mathrm{M} \Omega$, there appeared to be some difference in their distribution between $\mathrm{L}$ - and RG-elements. The effective resistance was not changed during activity and was independent of the change in ampli. tude of the response produced by the change in the intensity of illumination.

4) Based on the above results, it was fully discussed whether the nature of $S$-potential is of intra- or extracellular.

The authors wish to thank Dr. T. Tomita, Professor of Departmen of Physiology, Keio University, School of Medicine, for valuable discussion.

\section{REFERENCES}

1. Eccles, J. C. The Physiology of Nerve Cells. The Johns Hopkins Press, Baltimore, 1957.

2. GRUNDFEst, H. Electrical inexcitability of synapses and some consequences in the central nervous system. Physiol. Rev. 37: 337-361, 1957.

3. GRUNDFEST, H. cf. 6 .

4. LUNDBERG, A. Secretory potentials and secretion in sublingual gland of the cat. Nature, 177: 1080-1081, 1956.

5. MacNichol, E. F. Jr., Macpherson, L. AND Svaetichin, G. Studies on spectral response curves from the fish retina. Symp. on Visual Problems of Colour. Teddington, England, 1957.

6. MacNichol, E. F. JR. And Svaetichin, G. Electric responses from the isolated retinas of fishes. Am. J. Ophthal. 46: no. 3, Part II, 26-40, 1958.

7. Mitarai, G. The origin of the so-called cone potential. Proc. Japan Acadcmy. 34 : 299-304, 1958.

8. Oikawa, T., Ogawa, T. And Motokawa, K. Origin of so-called cone action potential. J. Neurophysiol. 22: 102-111, 1959.

9. Svaetichin, G. The cone action potential. Acta Physiol. Scana. 29: Suppl. 106: 565600, 1953. 
10. Tomita, T. A study on the origin of intraretinal action potential of the cyprinid fish by means of pencil-type microelectrode. Jap. J. Physiol. 7: 80-85, 1957.

11. Tomita, T., Tosaka, T., Watabe, K. And SAto, Y. The fish EIRG in response to different types of illumination, Jap. J. Physiol. 8: 41-50, 1958.

12. Tomita, T., Murakami, M., SAto, Y. and Hashimoto, Y. Further study on the origin of the so-called cone action potential ( $S$-potential). Its histological determination. Jap. J. Physiol. 9: 63-68, 1959.

13. WATANABE, K. AND TOSAKA, T. Functional organization of the cyprinid fish retina as revealed by discriminative responses to spectral illumination. Jap. J. Physiol. 9: 84-93, 1959. 